\title{
Potentially fewer minor complications with RARC than ORC but at what cost?
}

Robot-assisted radical cystectomy (RARC) is associated with a reduced risk of minor complications compared with open radical cystectomy (ORC), according to the largest comparative cohort study to date-involving 36,773 patients with bladder cancer surgically treated at 279 hospitals in the USA (34,672 and 2,101 of whom received RARC and ORC, respectively).

However, RARC was also associated with higher mean 90-day hospital costs than ORC (US\$31,007 vs $\$ 26,681$ ), even without accounting for the cost of the robotic platform-a finding that was largely attributed to the increased cost of supplies. Indeed, although patients who received RARC tended to stay in hospital for less time than those who received ORC (by a mean of 1.5 days), this outcome made little difference to room and board costs, presumably because most costs are associated with the initial part of hospitalization (intensive care).

Interestingly, when the authors restricted their analysis to only the highest-volume surgeons ( $\geq 7$ per year) and hospitals ( $\geq 19$ per year), no significant differences were observed between ORC and RARC with respect to morbidity or cost. The authors suggest that the similar costs at higher-volume centres could result from the fact that surgeons are further along the learning curve for RARC; alternatively, it could be that these centres use a streamlined postoperative care programme designed to minimize costs.

\section{Melanie Clyne}

Original article Leow, J. J. et al. Propensity-matched comparison of morbidity and costs of open and robot-assisted radical cystectomies: a contemporary population-based analysis in the United States. Eur. Urol. doi:10.1016/j.eururo.2014.01.029 